\title{
Polymorphisms of human vascular endothelial growth factor gene in high-altitude pulmonary edema susceptible subjects
}

\author{
Masayuki Hanaoka, ${ }^{1}$ Yunden Droma, ${ }^{1}$ Masao Ota, ${ }^{2}$ Michiko Ito, ${ }^{1}$ Yoshihiko Katsuyama ${ }^{3}$ \\ and Keishi Kubo ${ }^{1}$ \\ ${ }^{1}$ First Department of Medicine, Shinshu University School of Medicine, ${ }^{2}$ Department of \\ Legal Medicine, Shinshu University School of Medicine, ${ }^{3}$ Department of Pharmacy, \\ Shinshu University Hospital, Matsumoto, Japan
}

Correspondence:

Masayuki Hanaoka, MD, PhD

First Department of Medicine

Shinshu University School of Medicine

3-1-1 Asahi, Matsumoto 390-8621, Japan

Phone: +81-263-37-2631; Fax: +81-263-36-3722

E-mail: masayuki@hsp.md.shinshu-u.ac.jp 


\section{Authors' Contributions}

Hanaoka $M$ is the principal investigator of the study, and he obtained fund and supervised the study and helped to draft the manuscript. Droma Y contributed to the laboratory work and drafted the manuscript. Ota M supervised the study and helped to the statistical analyses. Ito $\mathrm{M}$ helped the laboratory work. Katsuyama $\mathrm{Y}$ helped the laboratory work and statistical analyses. Kubo K supervised the study. All authors read and approved the final manuscript.

\section{Summary at a Glance}

The single nucleotide polymorphisms (SNPs) of the vascular endothelial growth factor (VEGF) gene, namely C-2578A, G-1154A and T-460C in the promoter, G+405C in the 5'-untranslated region and C936T in the 3'-untranslated region of VEGF, were examined in 53 high-altitude pulmonary edema (HAPE) susceptible subjects (HAPE-s) and 69 HAPE resistant mountaineer controls (HAPE-r). This case-control study regarding the association of VEGF gene polymorphisms with the susceptibility to HAPE is expected to provide genetic evidence to shed light on the debate concerning the biological role of VEGF in the pathogenesis of HAPE. 


\begin{abstract}
Background and objective: Based on the reported biological property and function of vascular endothelial growth factor (VEGF) during hypoxic conditions, many investigations have studied the role of VEGF in the pathogenesis of high altitude sicknesses, including high-altitude pulmonary edema (HAPE). Unfortunately, no consistent findings have been demonstrated. We analyzed the association of VEGF gene polymorphisms with susceptibility to HAPE in order to shed light on the debate concerning the biological role of VEGF in the pathogenesis of HAPE.
\end{abstract}

Methods: The study included 53 HAPE susceptible subjects (HAPE-s) and 69 HAPE resistant mountaineer controls (HAPE-r). Subjects in both groups were Japanese and comparable in terms of age and sex ratios. The single nucleotide polymorphisms (SNPs) of the VEGF gene, namely C-2578A, G-1154A and T-460C in the promoter, G+405C in the 5'-untranslated region and C936T in the 3'-untranslated region of VEGF, were examined by allele discrimination experiments. In addition, arterial oxygen tension $\left(\mathrm{PaO}_{2}\right)$ and pulmonary hemodynamic data were available for 21 of the 53 HAPE-s subjects.

Results: No statistically significant differences were found in the allele frequencies, genotype distributions and haplotype frequencies of the examined SNPs of the VEGF 
gene between the HAPE-s and HAPE-r groups. Furthermore, neither the $\mathrm{PaO}_{2}$ nor the pulmonary hemodynamics was associated with the examined SNPs in the 21 HAPE-s subjects.

Conclusions: The current genetic study could not evidence that the functional SNPs of the VEGF gene are not associated with susceptibility to HAPE in a Japanese population.

Key words: high-altitude pulmonary edema, gene, permeability, polymorphism, vascular endothelial growth factor.

Short title: Polymorphisms of human VEGF gene in HAPE 


\section{INTRODUCTION}

High-altitude pulmonary edema (HAPE) is a non-cardiogenic pulmonary edema that occurs in non-acclimatized, previously healthy, and often young individuals within 2-4 days of rapid ascent above altitudes of 2,500 meters (m) above sea level. ${ }^{1}$ Although the mechanisms underlying the pathogenesis of HAPE are complex, the generally accepted paradigm of the pathogenesis of HAPE is related to the sequential process of high-altitude hypoxia-induced pulmonary hypertension, increased capillary permeability and compromise of the alveolar epithelial barrier, resulting in a high permeability pulmonary edema. ${ }^{2,3}$ Recurrent episodes are often reported, suggesting that individuals who have previously developed HAPE are more likely to experience future episodes than the general population. ${ }^{4}$ Thus, it has been speculated that a constitutional susceptibility determined by genetic background might underlie the development of this disease. $^{5}$

Vascular endothelial growth factor (VEGF) is shown to be significantly upregulated in the presence of hypoxia in rat $^{6,7}$ and human ${ }^{8}$ lungs. As well as a powerful angiogenic and endothelial-cell-specific mitogen, VEGF is a potent endothelial permeability factor expressed in most tissues, including lungs. ${ }^{9}$ Based on the reported biological property and function of VEGF under hypoxic conditions, ${ }^{10,11}$ many studies 
have analyzed the function of VEGF in the pathogenesis of high altitude sicknesses, including high-altitude cerebral edema (HACE), ${ }^{12}$ HAPE, ${ }^{13,14}$ and acute mountain sickness (AMS). ${ }^{15-18}$ However, high altitude studies regarding the altered systemic levels of VEGF in humans exposed to high altitude remain inconclusive; some authors have reported enhancing effects of hypoxic condition on VEGF blood concentrations, ${ }^{16,17}$ whereas others either found a lack of change ${ }^{13,15}$ or a decrease. ${ }^{14}$

The human VEGF gene is located on chromosome 6p21.3 and consists of eight exons that are alternatively spliced to generate isoenzymes of the VEGF protein. ${ }^{19}$ There is a considerable variation of correlation of the polymorphisms within the VEGF gene with the VEGF protein production, and analysis of the promoter region of VEGF gene has revealed the presence of many polymorphisms. ${ }^{20}$ Haplotype analyses of single nucleotide polymorphisms (SNPs) in the promoter and 5'-untranslated regions showed that the $-460 \mathrm{C}$ and $+405 \mathrm{C}$ alleles significantly alter VEGF promoter activity and responsiveness to biological stimuli. ${ }^{21}$ In addition, Prior et al. showed that the VEGF haplotype carrying the C-2578A, G-1154A, and G+405C SNPs impacted the enhancement of VEGF gene expression in human myoblasts under hypoxic conditions in vitro, which showed an association with maximal oxygen consumption in individuals before and after a standardized program of aerobic exercise training. ${ }^{22}$ Meanwhile, 
Renner et al. found that carriers of the 936T allele of the C936T SNP in the 3'-untranslated region of VEGF gene had significantly lower VEGF plasma levels than noncarriers. ${ }^{23}$ Therefore, we propose that analyzing the association of VEGF gene polymorphisms with susceptibility to HAPE could shed light on the debate regarding the biological role of VEGF in the pathogenesis of HAPE as described above. We hypothesized that the polymorphisms of VEGF gene might be associated with the susceptibility to HAPE. To address this association, five SNPs, including C-2578A, G-1154A and T-460C in the promoter, G+405C in the 5'-untranslated region and C936T in the 3'-untranslated region of VEGF, were examined in 53 HAPE susceptible subjects (HAPE-s) and 69 HAPE resistant subjects (HAPE-r) to investigate the association of these five SNPs with susceptibility to HAPE in a Japanese population.

\section{METHODS}

\section{Study subjects}

The case group consisted of 53 HAPE-s subjects, which included 46 males and 7 females with an average age of 34.2 years. All subjects had been hospitalized patients in the Shinshu University Hospital between July 1979 and September 2006 due to the onset of HAPE while climbing the Japan Alps $(2,758$ to $3,190 \mathrm{~m})$. The diagnosis of 
HAPE was based upon the criteria described previously. ${ }^{24}$ All subjects met the criteria of HAPE diagnosis at the onset of the disorder and recovered promptly with hospitalization. Examinations and cardiovascular tests were conducted in-hospital after their recovery to exclude any preexisting cardiopulmonary diseases.

The control group consisted of 69 elite mountaineers, including 59 males and 10 females, with an average age of 38.7 years. We defined these subjects as HAPE resistant subjects (HAPE-r) as they did not develop HAPE despite their exposure to a high-altitude environment over $3000 \mathrm{~m}$. All were elite mountaineers in the Mountaineering Association of Nagano Prefecture and the Alpine Club of Shinshu University, Japan. Subjects were given a questionnaire sheet that contained the components of Lake Louise Score ${ }^{25}$ during the recruitment, and no subject reported cardiopulmonary disorders and altitude-related problems while climbing mountains.

The HAPE-s and HAPE-r subjects were unrelated Japanese, born and residing in locations at heights of near sea level. HAPE-s and HAPE-r groups were comparable in terms of age, gender, ethnicity, and high-altitude exposure. This study and its investigational protocol were approved by the institutional ethics review board of Shinshu University for human study, and written informed consent was obtained from each case and control subject after a full explanation of the study. The procedures used 
in this human study were in accordance with the recommendations found in the Helsinki

Declaration. $^{26}$

\section{DNA extraction and genotyping assays}

Genomic DNA was extracted from venous blood by phenol extraction of sodium dodecyl sulfate lysed and proteinase $\mathrm{K}$ treated cells according to standard procedures. The final concentration of genomic DNA was prepared at 10-15 ng/ $\mu 1$ for the TaqMan SNP genotyping assays. The allelic discrimination of the VEGF gene polymorphisms was assessed with the Applied Biosystems 7500 Fast Real-time PCR System (Applied Biosystems, Foster City, CA, USA) using the fluorogenic 5' nuclease assay with TaqMan probes. SNP Genotyping Assay Mix for C-2578A (rs699947), G-1154A (rs1570360) and T-460C (rs833061), G+405C (rs2010963) and C936T (rs3025039) of VEGF gene (Figure 1) were obtained from Applied Biosystems (Tokyo, Japan).

\section{$\mathrm{PaO}_{2}$ and pulmonary hemodynamics in patients with HAPE}

The $\mathrm{PaO}_{2}$ was measured by standard laboratory methods on admission prior to oxygen therapy and right cardiac catheterization during breathing of room air was performed within 6 hours after admission to Shinshu University Hospital. A thermodilution Swan-Ganz catheter was introduced percutaneously into the pulmonary artery via the right internal jugular vein. The pulmonary artery pressure (PAP), pulmonary artery 
wedge pressure (PAWP), and cardiac output (CO) were measured. Pulmonary vascular resistance (PVR) was calculated by subtracting PAWP from PAP and dividing by the CO. The PVR was further corrected per square meter of body surface area and expressed as PVR index (PVRI).

\section{Statistical analysis}

Numerical values are presented as means \pm SD and categorical values are expressed as the number (n) and percentage (\%). Deviations from Hardy-Weinberg equilibrium were examined using the observed genotype frequency by the $\chi^{2}$ test. We assessed differences in the distributions of alleles and genotypes between the HAPE-s and HAPE-r groups by using the $\chi^{2}$ test. Haplotypes were inferred using the $\mathrm{R}$ package 'haplo.stats' in the $\mathrm{R}$ Project for Statistical Computing (http://www.r-project.org/). A haplotype was considered rare when its frequency was $1 \%$ or less. The differences in distributions of the detected haplotypes between the case and control groups were examined by the $\chi^{2}$ test. The sample size was estimated to be statistically sufficient to detect an association if the relative risk factor was 5 or greater with $80 \%$ power at the $5 \%$ significance level. In addition, the differences of the $\mathrm{PaO}_{2}$, mean PAP and PVRI in patients at the early stage of HAPE were analyzed by one-way analysis of variance (ANOVA) on variances of genotype and allele type. The criterion for statistical significance was $P<0.05$. 


\section{RESULTS}

\section{Genotypes of the VEGF gene in study subjects}

Hardy-Weinberg equilibrium was observed for all of the five examined SNPs in both cases and controls $(P>0.05)$. There were no significant differences in distributions of the genotypes and allele types for each of the SNPs of the VEGF gene between the HAPE-r and HAPE-s groups $(P>0.05$, Table 1$)$. The allele frequencies of the five VEGF SNPs in both the cases and controls were similar to those previously reported in a Japanese group $^{27}$ and another Asian population. ${ }^{28}$ Further comparisons of the frequencies of VEGF haplotypes for the five SNPs between the HAPE-r and HAPE-s are shown in Table 2. Nine patterns of haplotypes tagged by the five SNPs were identified in the HAPE-r group, and seven patterns were identified in the HAPE-s group. No statically significant differences were found in the frequencies of the VEGF haplotypes between cases and controls $(P>0.05$, Table 2$)$.

\section{Phenotype of the $\mathrm{PaO}_{2}$ and pulmonary hemodynamics in HAPE-s subjects}

The data for $\mathrm{PaO}_{2}$ and pulmonary hemodynamics at the early stage of disease were available for 21 of the 53 HAPE-s subjects because the examination of pulmonary hemodynamics was not performed in every patient with HAPE. The averages $\mathrm{PaO}_{2}$, 
mean PAP and PVRI were $40.9 \pm 9.6$ Torr, $28.4 \pm 8.1 \mathrm{mmHg}$ and $4.7 \pm 2.5$ $\mathrm{mmHg} \cdot \mathrm{L}^{-1} \cdot \mathrm{min}^{-1} \cdot \mathrm{m}^{-2}$, respectively, which were abnormal compared to the standard normal values of $>60 \mathrm{Torr},<25 \mathrm{mmHg}$ and $<2.5 \mathrm{mmHg} \cdot \mathrm{L}^{-1} \cdot \mathrm{min}^{-1} \cdot \mathrm{m}^{-2}$, respectively, in our hospital. The $\mathrm{PaO}_{2}$, mean PAP, and PVRI at early stage of disease did not show any significant differences between subgroups divided by the allele type of the tested SNPs in VEGF gene (Table 3).

\section{DISCUSSION}

We examined five SNPs of the VEGF gene that were suggested in previous studies to be functional in regulating VEGF mRNA in lung tissue and modifying VEGF concentrations in systemic circulation. ${ }^{20-23,27,28}$ We did not observe association of any of the five SNPs of VEGF with HAPE susceptibility in either genotypes or allele types. Correspondingly, no association between the identified haplotypes and susceptibility to HAPE was found. Moreover, the $\mathrm{PaO}_{2}$ and pulmonary hemodynamics data at the onset of HAPE were not associated with any of the examined SNPs in HAPE-s subjects.

The subjects in both the case and control groups were all Japanese, thus believed to be a homogenous population without population stratification. The allele frequencies of the five VEGF SNPs in both groups in the current investigation were similar to those 
reported previously in another Japanese group. ${ }^{27}$ In addition, the present study used the allelic discrimination assay to demonstrate allelic discrimination with a computerized system. This genotyping system permits the analysis of DNA samples with high sample-to-sample reproducibility. The confidence level of the automated allele calls was reported to be $99.7 \%$. Therefore, we consider that the experimental results are reliable and valid.

Acute hypoxia increases gene expressions of VEGF and its receptor in lung tissue. ${ }^{6,7,10,29}$ Hypoxia promotes oxidative base modifications in the promoter of VEGF gene in pulmonary artery endothelial cells. ${ }^{30}$ However, a recent human study revealed that, in contrary to in vitro studies, hypoxia decreases plasma VEGF concentrations in healthy humans, ${ }^{31}$ suggesting that systemic VEGF concentration might be regulated differently than its expression on the cellular basis. Although various in vitro studies have verified the impact of hypoxia on VEGF gene expression, no such hypoxic impact on circulating VEGF levels in humans has been found. High altitude studies regarding the altered systemic levels of VEGF in humans exposed to high altitude remain inconclusive; some authors have reported enhancing effects of hypoxic condition on VEGF blood concentrations, ${ }^{16,17}$ whereas others either found a lack of change ${ }^{13,15}$ or a decrease. ${ }^{14,31}$ In addition, Dorward, et al performed serial measurements of plasma 
VEGF levels in 38 healthy lowlanders at 3,650 m after 4-5 days of acclimatization and then 6 hours, 3 and 7 days after arriving at 5,200 m, and found an increase in plasma VEGF levels during acclimatization to high altitude. ${ }^{18}$ Their results did not support the conventional hypothesis that circulating unbound VEGF is an important component of the pathogenesis of acute mountain sickness, but rather suggested that VEGF is involved in acclimatization to high altitude. ${ }^{18}$ In our previous study, we also reported that the levels of VEGF in both the venous serum and bronchoalveolar lavage fluid of patients with HAPE were significantly higher during the recovery stage than upon admission to the hospital. ${ }^{14}$ We suspected that VEGF was probably destroyed in the lung of HAPE patients and it appeared less likely to have a critical function in the pathogenesis of HAPE, but rather an important role in the repair process for the impaired cell layer due to its biological functions in mitogen and angiogenesis. ${ }^{14}$ The current study provides genetic evidence in agreement with our previous proposal that VEGF does not have a pathological role in the pathogenesis of HAPE, but probably plays a part in recovering of HAPE or acclimatization to high altitude.

Evidence is accumulating to prove that HAPE is a pressure-induced leak pulmonary edema. $^{32}$ HAPE is thought to be caused by exaggerated hypoxic pulmonary vasoconstriction associated with decreased bioavailability of nitric oxide in the lungs. ${ }^{33}$ 
The inhomogenous exaggerated hypoxic pulmonary vasoconstriction ${ }^{34}$ can cause elevated pulmonary capillary pressure, ${ }^{35}$ which leads to high pressure and flow in affected areas, consequent mechanical over-distention of pulmonary capillaries, and subsequent injury of the blood-gas barriers. ${ }^{36}$ This pathogenesis causes extravasation of fluid, plasma proteins, and blood cells into the interstitial and alveolar spaces. ${ }^{37}$ All these studies ${ }^{32-37}$ strongly suggest that the hypoxic pulmonary vasoconstriction other than capillary permeability is the critical primary contributor to the pathogenesis of HAPE, although the capillary permeability may exacerbate the pathological process of pulmonary edema. ${ }^{38}$ Whilst the biological function of VEGF has little involvement with pulmonary vasoconstriction but mainly plays a role in powerful angiogenic mitogen and endothelial permeability. Additionally, in our study the examined SNPs of VEGF gene were not associated with the $\mathrm{PaO}_{2}$, mean PAP and PVRI in the 21 patients with HAPE. In view of these considerations, it is not surprise that our results did not evidence any associations of the VEGF gene polymorphisms with the susceptibility to HAPE.

The genomic DNA was extracted from the whole blood in all of our subjects; however, the plasma was only collected in our recent subjects so that the plasma VEGF concentration was not available for all subjects in this study. So we were unable to examine the association of the VEGF gene SNPs with systemic circulating VEGF 
concentrations. Another limitation is that the present sample size is relatively small for a genetic case-control association study compared to other case-control studies for common diseases such as type 2 diabetes ${ }^{27}$ and breast cancer. ${ }^{28}$ However, HAPE is a rare disease that occurs only in susceptible people who are rapidly ascending to high altitudes. Recently, effective mountaineering education in Japan has assisted in alerting susceptible individuals to the early symptoms of HAPE (by AMS score ${ }^{25}$ ) and enabling them to decide to descend immediately if risky symptoms appear during mountaineering. The present sample size is the most we could obtain in our hospital at present time. We expect to extend the present study to examine the association of the SNPs of VEGF gene with systemic VEGF levels to confirm the results in a larger sample size in future study.

In conclusion, we found no evidence that these functional SNPs of the VEGF gene are associated with susceptibility to HAPE in a Japanese population. In addition, these SNPs of the VEGF gene are not associated with the decreased $\mathrm{PaO}_{2}$ and increased mean PAP and PVRI levels observed in patients with HAPE at the early stages of the disease. Although no relationship between the SNPs of VEGF gene and the susceptibility to HAPE was found, we could not exclude the possibility that VEGF plays an important role in the recovering of HAPE or the acclimatization to high altitude. 


\section{ACKNOWLEDGMENTS}

This study was supported in part by a Grant-in-Aid for Scientific Research (C), No.

19590887, from the Japan Society for the Promotion of Science. We gratefully acknowledge Dr. Toshio Kobayashi for his support throughout the study and important comments to the manuscript. 


\section{REFERENCES}

1. Hackett PH, Roach RC. High-altitude illness. N. Engl. J. Med. 2001; 345: 107-114.

2. Gibbs JS. Pulmonary hemodynamics: implications for high altitude pulmonary edema (HAPE). A review. Adv. Exp. Med. Biol. 1999; 474: 81-91.

3. Hackett P, Rennie D. High-altitude pulmonary edema. JAMA 2002; 287: 2275-2278.

4. Hanaoka M, Kubo K, Yamazaki Y, Miyahara T, Matsuzawa Y, et al. Association of high-altitude pulmonary edema with the major histocompatibility complex. Circulation 1998; 97: 1124-1128.

5. Mortimer H, Patel S, Peacock AJ. The genetic basis of high-altitude pulmonary oedema. Pharmacol. Ther. 2004; 101: 183-192.

6. Christou H, Yoshida A, Arthur V, Morita T, Kourembanas S. Increased vascular endothelial growth factor production in the lungs of rats with hypoxia-induced pulmonary hypertension. Am. J. Respir. Cell Mol. Biol. 1998; 18: 768-776.

7. Levy AP, Levy NS, Wegner S, Goldberg MA. Transcriptional regulation of the rat vascular endothelial growth factor gene by hypoxia. J. Biol. Chem. 1995; 270: 13333-13340.

8. Fehrenbach H, Kasper M, Haase M, Schuh D, Muller M. Differential immunolocalization of VEGF in rat and human adult lung, and in experimental rat 
lung fibrosis: light, fluorescence, and electron microscopy. Anat. Rec. 1999; 254: $61-73$

9. Krenn K, Klepetko W, Taghavi S, Paulus P. Vascular endothelial growth factor increases pulmonary vascular permeability in cystic fibrosis patients undergoing lung transplantation. Eur. J. Cardiothorac. Surg. 2007; 32: 35-41.

10. Pham I, Uchida T, Planes C, Ware LB, Kaner R et al. Hypoxia upregulates VEGF expression in alveolar epithelial cells in vitro and in vivo. Am. J. Physiol. Lung Cell Mol. Physiol. 2002; 283: L1133-1142.

11. Pages G, Pouyssegur J. Transcriptional regulation of the vascular endothelial growth factor gene--a concert of activating factors. Cardiovasc. Res. 2005; 65: 564-573.

12. Xu F, Severinghaus JW. Rat brain VEGF expression in alveolar hypoxia: possible role in high-altitude cerebral edema. J. Appl. Physiol. 1998; 85: 53-57.

13. Pavlicek V, Marti HH, Grad S, Gibbs JS, Kol C et al. Effects of hypobaric hypoxia on vascular endothelial growth factor and the acute phase response in subjects who are susceptible to high-altitude pulmonary oedema. Eur. J. Appl. Physiol. 2000; 81: 497-503.

14. Hanaoka M, Droma Y, Naramoto A, Honda T, Kobayashi T, Kubo K. Vascular endothelial growth factor in patients with high-altitude pulmonary edema. J. Appl. 
Physiol. 2003; 94: 1836-1840.

15. Maloney J, Wang D, Duncan T, Voelkel N, Ruoss S. Plasma vascular endothelial growth factor in acute mountain sickness. Chest 2000; 118: 47-52.

16. Walter R, Maggiorini M, Scherrer U, Contesse J, Reinhart WH. Effects of high-altitude exposure on vascular endothelial growth factor levels in man. Eur. J. Appl. Physiol. 2001; 85: 113-117.

17. Tissot van Patot MC, Leadbetter G, Keyes LE, Bendrick-Peart J, Beckey VE et al. Greater free plasma VEGF and lower soluble VEGF receptor-1 in acute mountain sickness. J. Appl. Physiol. 2005; 98: 1626-1629.

18. Dorward DA, Thompson AA, Baillie JK, MacDougall M, Hirani N. Change in plasma vascular endothelial growth factor during onset and recovery from acute mountain sickness. Respir. Med. 2007; 101: 587-594.

19. Vincenti V, Cassano C, Rocchi M, Persico G. Assignment of the vascular endothelial growth factor gene to human chromosome 6p21.3. Circulation 1996; 93: 1493-1495.

20. Watson CJ, Webb NJ, Bottomley MJ, Brenchley PE. Identification of polymorphisms within the vascular endothelial growth factor (VEGF) gene: correlation with variation in VEGF protein production. Cytokine 2000; 12: 
1232-1235.

21. Stevens A, Soden J, Brenchley PE, Ralph S, Ray DW. Haplotype analysis of the polymorphic human vascular endothelial growth factor gene promoter. Cancer Res. 2003; 63: 812-816.

22. Prior SJ, Hagberg JM, Paton CM, Douglass LW, Brown MD et al. DNA sequence variation in the promoter region of the VEGF gene impacts VEGF gene expression and maximal oxygen consumption. Am. J. Physiol. Heart Circ. Physiol. 2006; 290: H1848-1855.

23. Renner W, Kotschan S, Hoffmann C, Obermayer-Pietsch B, Pilger E. A common $936 \mathrm{C} / \mathrm{T}$ mutation in the gene for vascular endothelial growth factor is associated with vascular endothelial growth factor plasma levels. J. Vasc. Res. 2000; 37: 443-448.

24. Kobayashi T, Koyama S, Kubo K, Fukushima M, Kusama S. Clinical features of patients with high-altitude pulmonary edema in Japan. Chest 1987; 92: 814-821.

25. Bartsch P, Roach R. Acute mountain sickness and high-altitude cerebral edema. In: Hornbein TF, Schoene RB, ed. High altitude: an exploration of human adaptation. New York: Basel: Marcel Dekker, 2001; 731-776.

26. World Medical Association Declaration of Helsinki: ethical principles for medical 
research involving human subjects. JAMA 2000; 284: 3043-3045.

27. Awata $\mathrm{T}$, Inoue $\mathrm{K}$, Kurihara $\mathrm{S}$, Ohkubo $\mathrm{T}$, Watanabe $\mathrm{M}$ et al. A common polymorphism in the 5'-untranslated region of the VEGF gene is associated with diabetic retinopathy in type 2 diabetes. Diabetes 2002; 51: 1635-1639.

28. Kataoka N, Cai Q, Wen W, Shu XO, Jin F et al. Population-based case-control study of VEGF gene polymorphisms and breast cancer risk among Chinese women. Cancer Epidemiol. Biomarkers Prev. 2006; 15: 1148-1152.

29. Tuder RM, Flook BE, Voelkel NF. Increased gene expression for VEGF and the VEGF receptors KDR/Flk and Flt in lungs exposed to acute or to chronic hypoxia. Modulation of gene expression by nitric oxide. J. Clin. Invest. 1995; 95: 1798-1807. 30. Grishko V, Solomon M, Breit JF, Killilea DW, Ledoux SP et al. Hypoxia promotes oxidative base modifications in the pulmonary artery endothelial cell VEGF gene. Faseb J. 2001; 15: 1267-1269.

31. Oltmanns KM, Gehring H, Rudolf S, Schultes B, Hackenberg C et al. Acute hypoxia decreases plasma VEGF concentration in healthy humans. Am. J. Physiol. Endocrinol. Metab. 2006; 290: E434-439.

32. Dehnert C, Berger MM, Mairbaurl H, Bartsch P. High altitude pulmonary edema: a pressure-induced leak. Respir. Physiol. Neurobiol. 2007; 158: 266-273. 
33. Duplain H, Sartori C, Lepori M, Egli M, Allemann Y et al. Exhaled nitric oxide in high-altitude pulmonary edema: role in the regulation of pulmonary vascular tone and evidence for a role against inflammation. Am. J. Respir. Crit. Care Med. 2000; 162: $221-224$.

34. Hanaoka M, Tanaka M, Ge RL, Droma Y, Ito A et al. Hypoxia-induced pulmonary blood redistribution in subjects with a history of high-altitude pulmonary edema. Circulation 2000; 101: 1418-1422.

35. Maggiorini M, Melot C, Pierre S, Pfeiffer F, Greve I et al. High-altitude pulmonary edema is initially caused by an increase capillary pressure. Circulation 2001; 103 : 2078-2083.

36. West JB, Tsukimoto K, Mathieu-Costello O, Prediletto R. Stress failure in pulmonary capillaries. J. Appl. Physiol. 1991; 70: 1731-1742.

37. Swenson ER, Maggiorini M, Mongovin S, Gibbs JS, Greve I et al. Pathogenesis of high-altitude pulmonary edema: inflammation is not an etiologic factor. JAMA 2002; 287: 2228-2235.

38. Robert JK, Ronald GC. Pathogenesis of high altitude pulmonary edema: Does alveolar epithelial lining fluid vascular endothelial growth factor exacerbate capillary leak? High. Alt. Med. Biol. 2004, 5: 399-409. 


\section{FIGURE LEGEND}

Figure 1 Structure of VEGF gene. The human VEGF gene is located on chromosome $6 \mathrm{p} 21.3$ and consists of eight exons. The arrow indicates the position of the single nucleotide polymorphism (SNP) examined in the current study. The solid box indicates exon and the opened box indicates intron, with the numeral indicating the length in base pair (bp). The numeral on dot-line indicates the length (bp) between two tested SNPs. 
Table 1 Distributions of the allele types and genotypes of the single nucleotide polymorphisms of VEGF gene in HAPE-r and HAPE-s subjects

Genotype or Allele type HAPE-r $(\mathrm{n}=69) \quad$ HAPE-s $(\mathrm{n}=53) \quad P$ value*

rs699947, promoter

$-2578 \mathrm{CC}$

$30(43.5 \%) \quad 27(50.9 \%) \quad 0.686$

$-2578 \mathrm{CA}$

$34(49.3 \%) \quad 22(41.5 \%)$

$-2578 \mathrm{AA}$

$5(7.2 \%) \quad 4(7.6 \%)$

$-2578 \mathrm{C}$

$94(68.1 \%)$

$76(71.7 \%)$

0.546

$-2578 \mathrm{~A}$

$44(31.9 \%) \quad 30(28.3 \%)$

rs1570360, promoter

$-1154 G G$

$50(72.5 \%)$

$39(73.6 \%) \quad 0.939$

$-1154 \mathrm{GA}$

$17(24.6 \%)$

$12(22.6 \%)$

$-1154 \mathrm{AA}$

$2(2.9 \%)$

$2(3.8 \%)$

$-1154 G$

$117(84.8 \%)$

$90(84.9 \%)$

0.979

$-1154 \mathrm{~A}$

$21(15.2 \%)$

$16(15.1 \%)$

rs833061, promoter

$-460 \mathrm{TT}$

$30(43.5 \%)$

$27(50.9 \%) \quad 0.686$

$-460 \mathrm{TC}$

$34(49.3 \%) \quad 22(41.5 \%)$ 


\begin{tabular}{|c|c|c|c|}
\hline$-460 \mathrm{CC}$ & $5(7.2 \%)$ & $4(7.6 \%)$ & \\
\hline$-460 \mathrm{~T}$ & $94(68.1 \%)$ & $76(71.7 \%)$ & 0.546 \\
\hline$-460 \mathrm{C}$ & $44(31.9 \%)$ & $30(28.3 \%)$ & \\
\hline \multicolumn{4}{|c|}{ rs2010963, 5'-UTR } \\
\hline$+405 \mathrm{GG}$ & $24(34.8 \%)$ & $13(24.5 \%)$ & 0.198 \\
\hline$+405 \mathrm{GC}$ & $36(52.2 \%)$ & $27(51.0 \%)$ & \\
\hline$+405 \mathrm{CC}$ & $9(13.0 \%)$ & $13(24.5 \%)$ & \\
\hline$+405 \mathrm{G}$ & $84(60.9 \%)$ & $53(50 \%)$ & 0.089 \\
\hline$+405 \mathrm{C}$ & $54(39.1 \%)$ & $53(50 \%)$ & \\
\hline \multicolumn{4}{|c|}{ rs3025039, 3'-UTR } \\
\hline $936 \mathrm{CC}$ & $42(60.9 \%)$ & $35(66.0 \%)$ & 0.770 \\
\hline $936 \mathrm{CT}$ & $25(36.2 \%)$ & $16(30.2 \%)$ & \\
\hline 936TT & $2(2.9 \%)$ & $2(3.8 \%)$ & \\
\hline $936 \mathrm{C}$ & $109(79.0 \%)$ & $86(81.1 \%)$ & 0.678 \\
\hline $936 \mathrm{~T}$ & $29(21.0 \%)$ & $20(18.9 \%)$ & \\
\hline
\end{tabular}

HAPE-r, high-altitude pulmonary edema resistant subjects; HAPE-s, HAPE susceptible subjects; UTR, untranslated region; VEGF, vascular endothelial growth factor.

$* P$ values (two-tailed) were calculated using $\chi^{2}$ test with $3 \times 2$ contingency table 
(genotype: $\mathrm{df}=2$ ) or $2 \times 2$ contingency table (allele type: $\mathrm{df}=1$ ). 
Table 2 Frequencies of haplotypes tagged by the five polymorphisms of VEGF gene in HAPE-r and HAPE-s subjects

\begin{tabular}{|c|c|c|c|c|c|c|c|c|}
\hline No. & rs699947 & rs 1570360 & rs833061 & rs 2010963 & rs3025039 & \multicolumn{2}{|c|}{ Frequency } & $P$ \\
\hline & $-2578 C>A$ & $-1154 \mathrm{G}>\mathrm{A}$ & $-460 \mathrm{~T}>\mathrm{C}$ & $+405 \mathrm{G}>\mathrm{C}$ & $936 \mathrm{C}>\mathrm{T}$ & HAPE-r & HAPE-s & value* \\
\hline$\# 1$ & $\mathrm{C}$ & $\mathrm{G}$ & $\mathrm{T}$ & $\mathrm{C}$ & $\mathrm{C}$ & 0.294 & 0.395 & 0.098 \\
\hline$\# 2$ & $\mathrm{C}$ & G & $\mathrm{T}$ & $\mathrm{G}$ & $\mathrm{C}$ & 0.265 & 0.217 & 0.387 \\
\hline \#3 & A & $\mathrm{G}$ & $\mathrm{C}$ & $\mathrm{G}$ & $\mathrm{C}$ & 0.138 & 0.090 & 0.248 \\
\hline$\# 4$ & $\mathrm{C}$ & $\mathrm{G}$ & $\mathrm{T}$ & $\mathrm{C}$ & $\mathrm{T}$ & 0.098 & 0.105 & 0.857 \\
\hline$\# 5$ & A & A & $\mathrm{C}$ & $\mathrm{G}$ & $\mathrm{C}$ & 0.085 & 0.110 & 0.511 \\
\hline$\# 6$ & A & $\mathrm{A}$ & $\mathrm{C}$ & $\mathrm{G}$ & $\mathrm{T}$ & 0.058 & 0.041 & 0.548 \\
\hline$\# 7$ & $\mathrm{~A}$ & $\mathrm{G}$ & $\mathrm{C}$ & $\mathrm{G}$ & $\mathrm{T}$ & 0.038 & 0.042 & 0.874 \\
\hline$\# 8$ & $\mathrm{C}$ & $\mathrm{G}$ & $\mathrm{T}$ & G & $\mathrm{T}$ & 0.016 & 0 & 0.191 \\
\hline$\# 9$ & $\mathrm{C}$ & A & $\mathrm{T}$ & $\mathrm{G}$ & $\mathrm{C}$ & 0.009 & 0 & 0.327 \\
\hline
\end{tabular}

HAPE-r, high-altitude pulmonary edema resistant subjects; HAPE-s, HAPE susceptible subjects; VEGF, vascular endothelial growth factor.

$* P$ values (two-tailed) were calculated using $\chi^{2}$ test with $2 \times 2$ contingency table $(\mathrm{df}=$ 1). 
Table 3 Associations of the $\mathrm{PaO}_{2}$, mean PAP and PVRI at the early stage of HAPE with the allele types of the SNPs of VEGF gene in 21 HAPE-s subjects

\begin{tabular}{|c|c|c|c|}
\hline \multirow[t]{2}{*}{ Allele type } & \multirow{2}{*}{$\begin{array}{l}\mathrm{PaO}_{2} \\
\text { (Torr) }\end{array}$} & \multirow{2}{*}{$\begin{array}{l}\text { Mean PAP } \\
(\mathrm{mmHg})\end{array}$} & \multirow{2}{*}{$\begin{array}{c}\text { PVRI } \\
\left(\mathrm{mmHg} \cdot \mathrm{L}^{-1} \cdot \mathrm{min}^{-1} \cdot \mathrm{m}^{-2}\right)\end{array}$} \\
\hline & & & \\
\hline \multicolumn{4}{|c|}{ rs699947, promoter } \\
\hline$-2578 \mathrm{C}$ & $42.17 \pm 10.46$ & $27.1 \pm 4.3$ & $4.19 \pm 2.08$ \\
\hline$-2578 \mathrm{~A}$ & $41.79 \pm 12.30$ & $28.4 \pm 3.97$ & $4.90 \pm 2.18$ \\
\hline$P$ value* & 0.93 & 0.43 & 0.42 \\
\hline \multicolumn{4}{|c|}{ rs1570360, promoter } \\
\hline$-1154 \mathrm{G}$ & $42.13 \pm 10.52$ & $27.2 \pm 4.3$ & $4.13 \pm 2.07$ \\
\hline$-1154 \mathrm{~A}$ & $37.88 \pm 10.84$ & $27.4 \pm 3.2$ & $4.53 \pm 1.66$ \\
\hline$P$ value* & 0.36 & 0.89 & 0.41 \\
\hline \multicolumn{4}{|c|}{ rs833061, promoter } \\
\hline$-460 \mathrm{~T}$ & $42.17 \pm 10.46$ & $27.1 \pm 4.3$ & $4.19 \pm 2.08$ \\
\hline$-460 \mathrm{C}$ & $41.21 \pm 12.79$ & $28.4 \pm 3.9$ & $4.90 \pm 2.18$ \\
\hline$P$ value* & 0.83 & 0.43 & 0.42 \\
\hline \multicolumn{4}{|c|}{ rs2010963, 5'-UTR } \\
\hline$+405 \mathrm{G}$ & $40.60 \pm 11.13$ & $27.3 \pm 4.3$ & $4.15 \pm 2.01$ \\
\hline
\end{tabular}


$P$ value*

0.44

0.43

0.53

rs3025039, 3'-UTR

936C

$41.42 \pm 10.38$

$27.2 \pm 4.2$

$4.15 \pm 2.01$

936T

$43.73 \pm 9.12 \quad 28.2 \pm 4.9$

$5.16 \pm 2.79$

$P$ value*

0.57

0.69

0.48

HAPE, high-altitude pulmonary edema; HAPE-s, HAPE susceptible subjects; $\mathrm{PaO}_{2}$, partial pressure of oxygen in arterial blood; PAP, pulmonary artery pressure; PVRI, pulmonary vascular resistance index; SNPs, single nucleotide polymorphisms; UTR, untranslated region; VEGF, vascular endothelial growth factor.

* $P$ values (two-tailed) were calculated using analysis of variance (ANOVA). 